\title{
Development of acute kidney injury following pediatric cardiac surgery
}

\author{
Aditya Sharma $^{1, *(\mathbb{1})}$, Ronith Chakraborty ${ }^{2, *}$, Katyayini Sharma ${ }^{3 \mathbb{D}}$, Sidharth K. Sethi ${ }^{4 \mathbb{D}}$, Rupesh Raina ${ }^{2,5}$ \\ 'Department of Internal Medicine, Northeast Ohio Medical University, Rootstown, OH, USA \\ 2Department of Nephrology, Cleveland Clinic Akron General Medical Center/Akron Nephrology Associates, Akron, OH, USA \\ ${ }^{3}$ Department of Medicine, DeBusk College of Osteopathic Medicine, Lincoln Memorial University, Harrogate, TN, USA \\ ${ }^{4}$ Department of Pediatric Nephrology and Pediatric Kidney Transplantation, Kidney and Urology Institute, Medanta, The Medicity \\ Hospital, Gurgaon, India \\ ${ }^{5}$ Department of Nephrology, Akron Children's Hospital, Akron, OH, USA
}

Acute kidney injury (AKI) in the pediatric population is a relatively common phenomenon. Specifically, AKI has been found in increasing numbers within the pediatric population following cardiac surgery, with up to $43 \%$ of pediatric patients developing AKI post-cardiac surgery. However, recent advances have allowed for the identification of risk factors. These can be divided into preoperative, intraoperative, and postoperative factors. Although the majority of pediatric patients developing AKI after cardiac surgery completely recover, this condition is associated with worse outcomes. These include fluid overload and increased mortality and result in longer hospital and intensive care unit stays. Detecting the presence of AKI has advanced; use of relatively novel biomarkers, including neutrophil gelatinase associated lipocalin, has shown promise in detecting more subtle changes in kidney function when compared to conventional methods. While a single, superior treatment has not been elucidated yet, novel functions of medications, including fenoldopam, theophylline and aminophylline, have been shown to have better outcomes for these patients. With the recent advances in identification of risk factors, outcomes, diagnosis, and management, the medical community can further explain the complexities of AKI in the pediatric population post-cardiac surgery.

Keywords: Acute kidney injury, Nephrology, Pediatrics, Thoracic surgery

Received April 11, 2020; Revised May 13, 2020;

Accepted May 13, 2020

Editor: Kent Doi, The University of Tokyo, Tokyo, Japan

Correspondence: Rupesh Raina

Consultant Nephrologist, Adult-Pediatric Kidney Disease/ Hypertension, Department of Nephrology, Cleveland Clinic Akron General and Akron Children's Hospital, 224 West Exchange Street, Akron,OH 44320, USA. E-mail: rraina@akronchildrens.org

*Aditya Sharma and Ronith Chakraborty contributed equally to this study as first authors.

Copyright ( 2020 by The Korean Society of Nephrology

(a) This is an open-access article distributed under the terms of the Creative Commons Attribution Non-Commercial License (http://creativecommons. org/licenses/by-nc-nd/4.0/), which permits unrestricted non-commercial use, distribution, and reproduction in any medium, provided the original work is properly cited.

\section{Introduction}

Cardiac surgery in the pediatric population is performed for a variety of reasons, including correction of congenital heart disease and acquired cardiac conditions. Pediatric cardiac surgery is not, however, without its risks, as post-operative complications have been seen in up to $43 \%$ of cases [1]. Among the risks is the development of acute kidney injury (AKI), a relatively common complication that can arise in the presence of multiple risk factors. Although AKI's exact mechanism, AKI's longterm outcomes, and treatment of AKI have yet to be fully illuminated, there have been advancements on these topics. Here, we describe the current understanding and practices as well as advances on the incidence, risk fac- 
tors, outcomes, biomarkers, and management of AKI in the pediatric population post-cardiac surgery.

\section{Incidence}

Postoperative AKI has been described to occur in $5 \%$ to $42 \%$ of pediatric patients undergoing cardiac surgery with mortality rates ranging from $20 \%$ to $79 \%$ [2]. The relatively wide range of AKI incidence can be partially accounted for by differences in the definition of AKI. For example, a larger study completed by Pederson et al [3] found the incidence of AKI among pediatric cardiac surgery patients to be $11.2 \%$. The requirement of dialysis was the marker for AKI in this study. However, only those with advanced AKI typically require dialysis; this cutoff may have missed cases of less severe AKI. In contrast, Park et al [4] used the Kidney Disease Improving Global Outcomes (KDIGO) criteria for AKI, a preferred, standardized definition, and found the incidence to be $41.8 \%$ among children and neonates undergoing cardiac surgery. Similarly, Zappitelli et al [5] found the incidence of AKI to be $36 \%$. In addition to the definition of AKI used, other factors such as baseline characteristics, complexities of cardiac condition, and surgeries performed may all have contributed to the variation in incidence of AKI. With the potential to develop AKI in up to $42 \%$ of patients, the vulnerable population should be closely monitored to prevent advancement of AKI.

\section{Pathophysiology}

The primary mechanisms through which AKI develops when associated with cardiac surgery include renal ischemia, reperfusion injury, inflammation, and cardiopulmonary bypass (CPB)-induced hemolysis (Fig. 1) [6]. The nature of the blood supply in the kidney, in which the majority of blood is filtered by cortical glomeruli, inherently increases risk of ischemia to the renal medulla. Furthermore, the slow movement of blood through the vasa recta, the primary blood supply for the medulla, compounds the risk of ischemia to the renal medulla [7]. Therefore, in situations of decreased perfusion pressures, such as during cardiac surgery, the risk of renal medullary hypoxia and associated AKI increases. Exacerbating the problem, the reperfusion that follows activates proinflammatory transcription factors, including nuclear factor kappa-B, and increases reactive oxygen species (ROS) development. This results in reperfusion injury $[8,9]$. The resulting inflammatory state, mediated by increased cytokines and chemokines, further damages renal tissue by recruiting neutrophils, lymphocytes, and macrophages into the renal parenchyma. This process can result in permanent renal damage due to the consequent healing and fibrosis [10].

In addition to the ischemic and inflammatory pathways, the use of CPB inherently increases the risk of developing AKI. CPB involves extracorporeal use of a mechanical pump in place of the heart; blood has to flow through the pump, oxygenator, and catheter compartments in order to circulate back to the body in an oxygenated manner. This increases the risk for mechanical damage and consequent hemolysis, which can lead to increased serum hemoglobin levels. Not only can the increased hemoglobin lead to further production of ROS and vasoconstriction of the renal arterioles, but this hemoglobin may also accumulate and precipitate in the renal tubules, causing pigment nephropathy and AKI $[11,12]$. In addition, extracorporeal exposure of blood has been shown to increase levels of interleukin (IL)-6, IL-8, and tumor necrosis factor- $\alpha$, further contributing to development of AKI [13].

\section{Risk factors}

The development of AKI in children undergoing cardiac

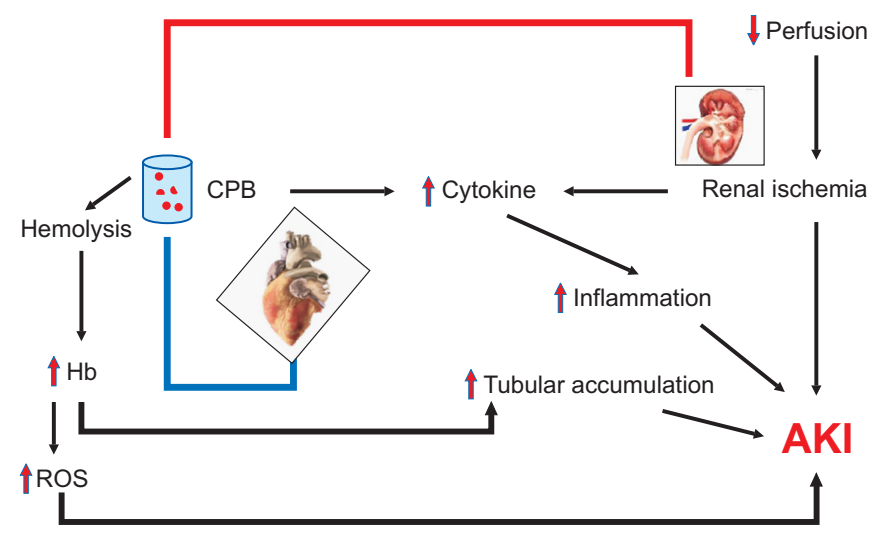

Figure 1. Pathogenesis of acute kidney injury (AKI) in cardiac surgery.

$\mathrm{CPB}$, cardiopulmonary bypass; Hb, hemoglobin; ROS, reactive oxygen species. 
surgery is often multifactorial. In this dynamic setting, the risk factors associated with development of AKI can be divided into three categories: preoperative, intraoperative, and postoperative (Table 1) [2,4,14-19].

\section{Preoperative}

Identifying the risk factors associated with development of AKI in pediatric cardiac surgery patients prior to surgical intervention provides an opportunity to detect and prevent complications earlier. Younger age is a risk factor; neonates have the highest rates of AKI [14]. Both Park et al [4] and Sethi et al [15] found age < 12 months to be significantly associated with increased risk of AKI. Similarly, Zappitelli et al [5] reported the median age of 1.1 years among those developing AKI. Li et al [2] identified children under age 2 years as having the highest rates of AKI; $67 \%$ of AKI events occurred in children between 30 days and 2 years of age in their study. This contrasts with the incidence of $28 \%$ and $5 \%$ seen in children 2 to 13 years old and over 13 years, respectively. Such a relationship may be related to immature renal parenchyma. The renal parenchyma probably fully matures by the age of 2 years and is more prone to ischemic and inflammatory injury prior to complete maturation [2].

Lower mean preoperative weight correlated with an increased risk of developing AKI. Li et al [2] found that those with a preoperative mean weight of $2.4 \mathrm{~kg}$ developed AKI more frequently than those who had a preoperative weight of $19 \mathrm{~kg}$. A retrospective analysis performed by Lee et al [16] demonstrated similar results; lower mean preoperative weight was associated with increased incidence of AKI. In addition, Lee et al [16] found preoperative mechanical ventilation to be associated with AKI.

However, there are conflicting findings on the influence of baseline serum creatinine on development of AKI in this study population. Blinder et al [14] found a higher reference serum creatinine to be significantly associated with postoperative AKI (odds ratio, 5.1; 95\% confidence interval $[\mathrm{CI}], 1.94-13.2 ; P$ value $=0.0009$ ). In contrast, $\mathrm{Li}$ et al [2] described a significant difference in preoperative serum creatinine levels, but the same was not seen on comparison of estimated glomerular filtration rates. The variability among baseline serum creatinine levels may be due to multiple factors including age, sex, muscle mass, and fluid homeostasis. The effect of baseline creatinine on development of AKI in the pediatric cardiac surgery patient population is still uncertain [14].

Table 1. Risk factors for development of acute kidney injury

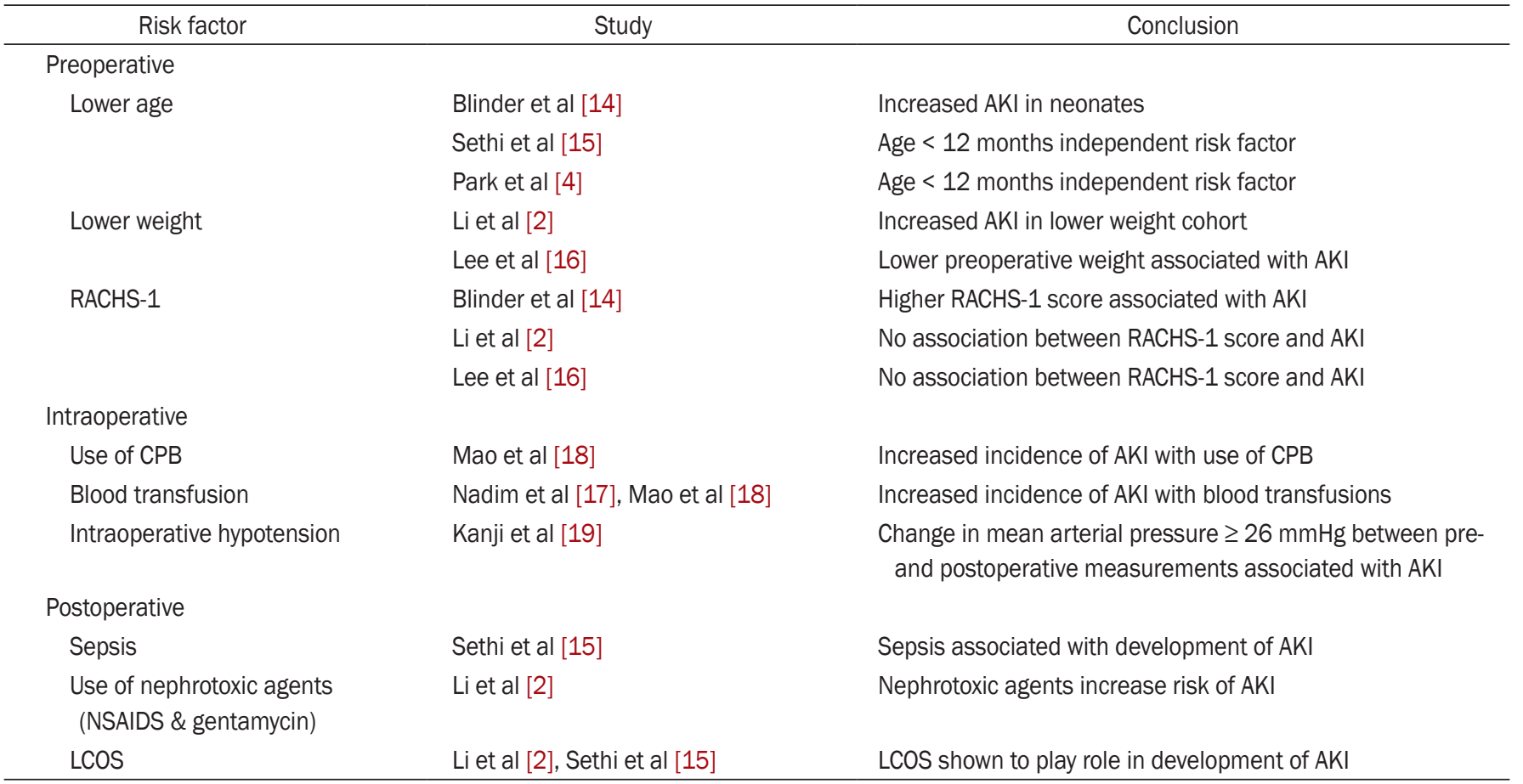

AKI, acute kidney injury; CPB, cardiopulmonary bypass; LCOS, low cardiac output syndrome; NSAIDS, non-steroidal anti-inflammatory drugs. 
Finally, there is some evidence that the complexity of cardiac surgery being performed, gauged by the RACHS-1 score, or risk-adjusted classification for congenital heart surgery, plays a role in the development of AKI, as seen by Blinder et al [14]. However, other studies have shown that RACHS-1 was not a significant factor in the development of AKI. This was demonstrated by the prospective analysis performed by Li et al [2] as well as other studies, including the retrospective analysis by Lee et al [16], all of which suggest that the complexity of cardiac operation being performed is not associated with the development of AKI. With the conundrum posed by the conflicting results of these studies, randomized controlled trials are required to explicate the true effect of increasing cardiac surgery complexity on the development of AKI.

\section{Intraoperative}

Many intraoperative factors have been implicated in the development of AKI $[2,17,18,20]$. These factors include use of $\mathrm{CPB}$, length of $\mathrm{CPB}$, blood transfusions, and intraoperative hypotension. Although CPB provides for continued perfusion of body organs during heart surgery, $\mathrm{CPB}$ use poses an inherent risk for remote organ damage including kidney damage $[19,20]$.

Aside from the inherent risk associated with any use of $\mathrm{CPB}$, the length of time in which a patient is on $\mathrm{CPB}$ has also been associated with increased risk of AKI. In a retrospective analysis, Blinder et al [14] found that $83 \%$ of infants who developed AKI post-cardiac surgery required CPB $(P<0.0001)$. Similarly, Li et al [2] found a significantly higher mean CPB time in patients who developed AKI versus those who did not and showed that use of $\mathrm{CPB}$ for $>180$ minutes was associated with 7.57 times higher odds of developing AKI versus those that required $<60$ minutes of CPB. Zappitelli et al [5] also reported higher odds of AKI with longer CPB time (odds ratio, 1.01; 95\% CI, 1.003-1.01) by multivariable logistic regression.

Like the mechanism behind CPB-mediated AKI, blood transfusions can also lead to AKI through inflammatory processes [17,18]. Zappitelli et al [5] showed higher rates of AKI in children who received larger amounts of blood intraoperatively. Similarly, Park et al [4] demonstrated a significant risk of AKI in those receiving a median value of $62.6 \mathrm{~mL} / \mathrm{kg}$ of blood versus those receiving $41.9 \mathrm{~mL} / \mathrm{kg}$. Park et al [4] also concluded that an increase of hemoglo- bin $>3 \mathrm{~g} / \mathrm{dL}$ postoperatively was associated with developing AKI. However, these researchers were not able to clearly delineate whether this increased risk of AKI was due to hemoconcentration, underlying cardiac physiology, or changes in hematocrit with CPB use.

Furthermore, intraoperative hypotension can lead to decreased end-organ perfusion and result in AKI [2]. Li et al [2] found that $88 \%$ of patients who developed AKI had hypotension (as defined by mean arterial pressure (MAP) $<50 \mathrm{mmHg}$, MAP $<40 \mathrm{mmHg}$ if $<1$ year old, or MAP $<50$ $\mathrm{mmHg}$ with inotropic support) for a mean of 75 minutes. In addition, Kanji et al [19] found that a change in MAP $\geq$ $26 \mathrm{mmHg}$ between preoperative and intraoperative measurements was associated with development of AKI in high-risk adults undergoing cardiac surgery (odds radio, 2.8). Therefore, maintaining adequate perfusion is crucial. This requires monitoring and managing the dynamic blood pressures according to each patient's presentation based on factors such as age, size, temperature, and presence of underlying heart disease and anatomical shunts $[17,20]$.

\section{Postoperative}

Even in the postoperative period, there are well-known factors that are associated with the development of AKI. These include sepsis and consequent use of nephrotoxic agents, low cardiac output syndrome (LCOS), and need for ventilatory support $[2,15,21-24]$.

Sepsis has been known to portend a poor prognosis in the postoperative period, not only due to the inherent nature of sepsis, but also due to the subsequent treatments that are used. Sethi et al [15] found a significantly higher level of sepsis in those that developed AKI than in those that did not ( $60 \%$ vs. $15 \%$ respectively). Similar results were reported by Alam et al [21], but a significant difference in the development of AKI in the sepsis group was not exhibited on multivariable analysis. In addition, treatment of sepsis can include use of antibiotics and nonsteroidal anti-inflammatory drugs (NSAIDS), some of which have nephrotoxic potential. Li et al [2] found that among patients with AKI, 15\% received gentamycin and $56 \%$ received NSAIDS for analgesia. Both gentamycin and NSAIDs can have a worsening effect in AKI. Therefore, use of drugs and dyes with nephrotoxic potential should be judicious in postoperative patients with high risk of 
AKI.

LCOS is a condition in which there is a decrease in bodily perfusion due to decreased cardiac output [22]. In the postoperative period following cardiac surgery, the etiology of LCOS is multifactorial. Inherent heart disease and myocardial stress play significant roles in LCOS development [23]. Li et al [2] identified LCOS in $6 \%$ of patients who developed AKI, and LCOS has also been shown to play a significant role in development of AKI. An odds ratio of 6.4 (95\% CI) for developing AKI in those with LCOS was found by Sethi et al [15]. Therefore, management of LCOS can be critical in preventing development of AKI. LCOS is initially medically managed; the goal is to maintain adequate end-organ perfusion. Medical management is accomplished with the use of catecholamines and inotropes (levosimendan) for ionotropic support and phosphodiesterase inhibitors (milrinone) for reduction of pulmonary and systemic vascular resistance. Steroids can be used in catecholamine-resistant cardiac failure, and extracorporeal life support is used when all medical management fails [24].

Like cardiac malfunction seen in LCOS, pulmonary dysfunction and consequent need for mechanical ventilation is also associated with development of AKI post-cardiac surgery. Li et al [2] found a 3.2 odds ratio of developing AKI in those needing mechanical ventilation vs. those who did not. Li et al [2] also found increasing duration of time on mechanical ventilation to be associated with increasing severity of AKI as defined by the pRIFLE criteria $(P=0.039)$. The need for mechanical ventilation may be related to the fluid-overloaded state and ensuing poor respiratory parameters; these can occur as an outcome of AKI.

\section{Outcomes}

Overall, nearly $90 \%$ of pediatric patients developing AKI within the post-cardiac surgery period completely recover by the fourth operative day [2]. However, the development of AKI in the post-cardiac surgery period has been found to be associated with multiple negative outcomes, including the fluid-overloaded state, longer hospital and intensive care unit (ICU) stays, and increased mortality [2,5,14,15,25-28].

A fluid-overloaded state can arise not only from aggressive fluid administration, LCOS, and capillary leak syndrome, but also from AKI [25]. While there is debate as to the cause-effect relationship between AKI and fluid overload, one aspect is certain: both are associated with worsened outcomes for patients [26]. An observational study and consequent secondary analysis performed by Hassinger et al [27] found that children with early fluid overload ( $\geq 5 \%$ at the end of postoperative day 1 ) had lower baseline eGFRs compared to those with normal fluid balance. While this research group did find an association between fluid overload and AKI, this was not a consistent finding within their own assessment. Similarly, Delpachitra et al [28] found no association between fluid overload and AKI in a case-controlled study performed to assess association.

Patients with AKI also have longer ICU and hospital stays when compared to those without AKI [2]. Li et al [2] found a significant difference in median hospital stay, 7 days for the AKI group and 4 days for the non-AKI group. Zappitelli et al [5] determined that the length of PICU stay was independently associated with AKI. In addition to longer hospital stays, AKI was found to be significantly associated with increased mortality. Alam et al [21] reported a 22.6 odds ratio of mortality for those at stage 2 AKI and an 18 odds ratio for those at stage 3 AKI. Similarly, Blinder et al [14] found a significantly higher mortality rate in the AKI group (11.6\%) than. in the non-AKI group (2.9\%).

Long-term outcomes in children who develop AKI have not been fully clarified. Although there have been studies exploring the long-term outcomes, no clinically significant associations have been established [2,29]. Cooper et al [30] discovered higher levels of common biomarkers used to identify AKI, including kidney injury molecule-1 (KIM-1), urinary liver type fatty acid binding protein (LFABP), and IL-18, at a mean of 7 years post-cardiac surgery, but there were no differences in the rates of chronic kidney disease found between the groups. The relative lack of long-term findings suggests the need for further studies into the long-term effects of AKI in the post-cardiac surgery period.

\section{Biomarkers}

Although often used in the diagnosis of AKI, serum creatinine is an imperfect marker for AKI and often arises later in the course of AKI [31]. In addition, many different 
factors affect creatinine levels, including body habitus, age, volume status, and nutrition. Other markers that allow for quicker identification of kidney injury have been discovered (Table 2) [32-37]. One relatively novel biomarker used to identify AKI is cystatin C. Cystatin C belongs to the cysteine protease inhibitor family and can reveal subtle damage to the glomerulus [32]. Zappitelli et al [38] found that increasing levels of cystatin C correlated with severity of AKI, with adjusted odds ratios of 6.4 and 20 for stage 1 and stage 2 AKI, respectively. Additionally, Peco-Antić et al [33] showed a rise in cystatin $\mathrm{C}$, in addition to urinary L-FABP, 2 hours post-use of CPB had superior accuracy for predicting AKI. These had area under receiver operating curves of 0.89 and 0.73 , respectively. This contrasts with the findings of Koyner et al [39] who failed to show similar positive associations between urinary cystatin $\mathrm{C}$ and AKI. Other biomarkers are useful predictors of AKI in the pediatric post-cardiac surgery population. These biomarkers include neutrophil gelatinase lipocalin (NGAL), a marker for tubular damage; tissue inhibitor of metalloproteinases-2; insulin-like growth factor-binding protein 7; and inflammatory markers such as IL-6 and IL-18 [34,39]. Basu et al [35] showed a composite of biomarkers, specifically the composite of NGAL and cystatin $\mathrm{C}$, was superior to the use of creatinine for detecting and predicting AKI. The NGAL and cystatin C composite demonstrated a positive likelihood ratio of severe AKI to be 10 times stronger than creatinine level. Also, the positive predictive value of severe AKI was twice as high when using this composite versus creatinine (88\% vs. $45 \%$, respectively). Randomized control trials, such as that of Meersch et al [40], have evaluated the levels of urinary biomarkers in the post-cardiac surgery period for adults. However, no studies have evaluated the applicability and usefulness of these biomarkers in pediatric post-cardiac surgery patients. Such studies are warranted.

\section{Management}

A single, superior treatment for AKI has yet to be resolved even though some treatments have been studied extensively. Novel methods of treatment to ameliorate kidney damage are also being studied (Table 3) [41]. The medical treatments available include diuretics, specifically furosemide. While influencing the physiology involved in AKI, furosemide does not aid in halting development and progression of AKI [42]. However, fenoldopam, a dopamine receptor agonist that has vasodilatory effects in the kidneys, has been found to provide benefit among infants who underwent CPB. Ricci et al [43] found that treatment of pediatric patients undergoing cardiac surgery with high-dose fenoldopam had a statistically significant association with decreased levels of urinary NGAL and cystatin C, two of the most accurate markers of AKI. Similarly, theophylline and aminophylline, both xanthine derivatives that inhibit adenosine-induced vasoconstriction, were found to potentially prevent the development of AKI [41]. Further studies are needed to evaluate the safety and efficacy of these drugs in the pediatric popula-

Table 2. Biomarkers of acute kidney injury

\begin{tabular}{lll}
\hline \multicolumn{1}{c}{ Biomarker } & \multicolumn{1}{c}{ Role of: } & \multicolumn{1}{c}{ Study } \\
\hline Cystatin C & Glomerular damage & Villa et al [32] \\
Neutrophil gelatinase-associated lipocalin & Tubular damage & Basu et al [35] \\
Tissue inhibitor of metalloproteinase 2 & Cell cycle arrest [36] & Yuan [34] \\
Insulin-like growth factor-binding protein 7 & Cell cycle arrest [36] & Yuan [34] \\
Interleukin-6 & Inflammation & Yuan [34] \\
Interleukin-18 & Inflammation & Yuan [34] \\
Liver type fatty acid binding protein & Tubular damage [37] & Peco-Antić et al [33] \\
\hline
\end{tabular}

Table 3. Medical management of acute kidney injury following cardiac surgery

\begin{tabular}{lll}
\hline \multicolumn{1}{c}{ Treatment } & \multicolumn{1}{c}{ Mechanism } & \multicolumn{1}{c}{ Action in kidney } \\
\hline Furosemide & $\mathrm{Na}^{+}, \mathrm{K}^{+}, 2 \mathrm{Cl}$ symporter (NKCC2) antagonist & Diuresis \\
Fenoldopam & $\mathrm{D} 1$ receptor antagonist & Renal vasodilation and increased blood flow \\
Theophylline \& aminophylline (xanthine derivatives) & Inhibit adenosine-induced vasoconstriction & Increased renal blood flow, diuresis \\
\hline
\end{tabular}


tion undergoing cardiac surgery.

The use of kidney replacement therapy (KRT) has become common practice in settings of fluid overload, electrolyte abnormalities, and AKI in pediatric patients undergoing cardiac surgery (Table 4) [44-47]. In such cases, peritoneal dialysis (PD) is preferred over other modalities of KRT because of the ease of venous access and placement of the catheter and the lack of need for anticoagulation therapy [41]. In addition, early initiation of PD has been associated with improved outcomes. Namachivayam et al [48] demonstrated that early initiation of PD was associated with lower incidence of cardiac arrest, lower need for emergency chest opening, lower requirement for extracorporeal membrane oxygenation, and lower mortality. Secondarily, shorter ICU stays and reduced ventilator use were required. Kwiatkowski et al [49] also showed that those patients receiving the PD catheter had a higher percentage of negative fluid balance, shorter time to negative fluid balance, earlier extubation, improved inotrope scores, and fewer electrolyte disturbances. In contrast, Ryerson et al [50]'s randomized control trial showed no benefits of initiating prophylactic PD. However, this study had a relatively small sample size of 22 neonates; larger, multi-center randomized controlled trials are needed to evaluate the true efficacy of early initiation of PD.

In cases of critically ill children, continuous renal replacement therapy (CRRT) is preferred. CRRT allows for both slow and controlled removal of fluid, avoiding significant changes in the patient's fluid balance [44]. However, in contrast to PD, CRRT can be difficult to implement due to the complexity of canalization and difficulty of catheter placement, further justifying the use of KRT in treating AKI and fluid overload in the pediatric postcardiac surgery population.

\section{Future directions: Diagnosis, risk stratification, and treatments}

The realm of AKI has been rapidly evolving in recent years with advancements in diagnosis and management. Diagnosis of AKI has continuously been refined with biomarkers detecting kidney injury earlier than ever before. However, recent findings have led to a shift in the paradigm from biomarkers detecting injury to biomarkers detecting the "pre-injury phase". These theorized preinjury phase biomarkers include microRNAs (miRNAs), hemojuvelin, and Dickkopf 3 (DKK3) transcription factor [51]. MicroRNAs, or single-stranded mRNAs that are theorized to have a role in gene regulation, appear to have paradoxical effects on both inflammation and apoptosis [52]. As such, further investigation of the role of miRNAs in development of AKI may allow for development of sensitive biomarkers and potential treatments. Hemojuvelin, a membrane protein involved in iron regulation, has been found in increased concentrations in the urine of patients with AKI and may prove to be an early marker of AKI [51]. Finally, DKK3, a stress-induced transcription factor that disrupts Wnt/beta-catenin signaling and may be involved in promoting tubular repair, could serve as a molecular sign of imminent injury to the kidney [53]. While these molecules show promise in detection of the pre-injury phase, their roles as biomarkers of AKI need to be thoroughly validated prior to use in clinical practice.

The ability to stratify patients based on severity of AKI has been a useful concept. Tools such as the Cleveland Clinic Score and Mehta Score allow for risk stratification for severe AKI, and the KDIGO criteria serve as risk stratification for mild-moderate AKI. However, a risk stratification technique using novel biomarkers is not available; creation of a classification system that incorporates novel and highly sensitive biomarkers would be greatly ben-

Table 4. Dialytic management of acute kidney injury following cardiac surgery

\begin{tabular}{llcc}
\hline \multicolumn{1}{c}{ Dialytic modality } & \multicolumn{1}{c}{ Indication } & Benefit & Limitations/risk \\
\hline Peritoneal dialysis (PD) & Fluid overload, mild-moderate AKI & $\begin{array}{c}\text { Ease of access, decreased time } \\
\text { to negative fluid balance }\end{array}$ & $\begin{array}{c}\text { Peritonitis, relatively decreased } \\
\text { filtration efficiency [45] }\end{array}$ \\
$\begin{array}{llcl}\text { Intermittent hemodialysis (IHD) } & \text { Fluid overload, AKI } & \text { Rapid fluid \& solute removal } & \text { Rapid fluid shift [46] } \\
\text { Continuous renal replacement } & \text { Critically-ill patients, severe AKI, } & \text { Avoidance of major fluid shifts; } & \text { Complexity of implementation [44] } \\
\text { therapy (CRRT) } & \text { fluid overload } & \text { controlled removal of fluid } & \\
\text { Continuous venovenous } & \text { Severe AKI \& fluid overload } & \text { Highly-efficient filtration method } & \text { Hypotension, hypothermia, } \\
\text { hemodiafiltration (CVVHDF) } & & \text { to remove both solutes \& fluids } & \text { bleeding [47] }\end{array}$ \\
\hline
\end{tabular}

AKI, acute kidney injury. 
eficial for accurately identifying at-risk patients for renal damage [6].

Within the recent past, there have been some exciting developments in the treatment of AKI. Novel treatments for AKI have been investigated, leading to the identification of mesenchymal stem cells and alkaline phosphatase as potential management options. Mesenchymal stem cells have anti-inflammatory properties that promote tissue repair and increase cell survival [6]. Hattori et al [54] found that injection of stem cells from human exfoliated deciduous teeth directly into the subcapsular renal space of mice undergoing induction of renal ischemia showed significantly lower serum cytokines, creatinine, and blood urea nitrogen levels when compared to the control group. Similarly, Herrera Sanchez et al [55] found that intravenous injection of human liver stem cells into mice undergoing induction of AKI significantly decreased serum creatinine and urea, hyaline cast formation, and tubular necrosis. In addition, mice injected with stem cells also showed greater tubular cell proliferation and regeneration. While no similar study has been completed in humans, there is currently an ongoing clinical trial evaluating the efficacy of stem cells in cisplatin-induced AKI (ClinicalTrials.gov NCT01275612). Alkaline phosphatase, an enzyme which converts pro-inflammatory adenosine triphosphate into anti-inflammatory adenosine, has also shown promise in AKI treatment [6]. Pickkers et al [56] found decreased levels of inflammatory markers, such as IL-6 and C-reactive protein, as well as decreased urinary excretion of KIM-1 and IL-18, in septic patients given intravenous infusion of alkaline phosphatase. With these potential treatments on the horizon, AKI may become a more manageable and less damaging disease in the near future.

\section{Conclusion}

The development of AKI in the pediatric population undergoing cardiac surgery is a common complication with as many as $42 \%$ of this patient population developing AKI [2]. An exact mechanism and a single, superior treatment have yet to be fully revealed. However, by identifying common risk factors, outcomes, and treatment modalities, both current and novel, the medical community will have a basis from which further large-scale studies can be performed in order to fully unravel the complexities of
AKI diagnosis and treatment in the pediatric population undergoing cardiac surgery.

\section{Conflicts of interest}

All authors have no conflicts of interest to declare.

\section{Authors' contributions}

Aditya Sharma and Ronith Chakraborty were involved in collecting data \& writing of manuscript. Katyayini Sharma and Sidharth K. Sethi were involved with synthesizing of manuscript. Rupesh Raina provided intellectual content and was involved in collecting data, conceptualization, design, and writing of manuscript.

\section{References}

[1] Agarwal HS, Wolfram KB, Saville BR, Donahue BS, Bichell DP. Postoperative complications and association with outcomes in pediatric cardiac surgery. $J$ Thorac Cardiovasc Surg 2014;148:609-616.

[2] Li S, Krawczeski CD, Zappitelli M, et al. Incidence, risk factors, and outcomes of acute kidney injury after pediatric cardiac surgery: a prospective multicenter study. Crit Care Med 2011;39:1493-1499.

[3] Pedersen KR, Povlsen JV, Christensen S, et al. Risk factors for acute renal failure requiring dialysis after surgery for congenital heart disease in children. Acta Anaesthesiol Scand 2007;51:1344-1349.

[4] Park SK, Hur M, Kim E, et al. Risk factors for acute kidney injury after congenital cardiac surgery in infants and children: a retrospective observational study. PLoS One 2016; 11:e0166328.

[5] Zappitelli M, Bernier PL, Saczkowski RS, et al. A small postoperative rise in serum creatinine predicts acute kidney injury in children undergoing cardiac surgery. Kidney Int 2009;76:885-892.

[6] O'Neal JB, Shaw AD, Billings FT 4th. Acute kidney injury following cardiac surgery: current understanding and future directions. Crit Care 2016;20:187.

[7] Brezis M, Rosen S. Hypoxia of the renal medulla--its implications for disease. N Engl J Med 1995;332:647-655.

[8] Wei C, Li L, Kim IK, Sun P, Gupta S. NF- $\kappa$ B mediated miR21 regulation in cardiomyocytes apoptosis under oxidative stress. Free Radic Res 2014;48:282-291. 
[9] Ali F, Sultana S. Repeated short-term stress synergizes the ROS signalling through up regulation of NFkB and iNOS expression induced due to combined exposure of trichloroethylene and UVB rays. Mol Cell Biochem 2012;360:133145.

[10] Schrier RW. Diseases of the kidney \& urinary tract. Philadelphia: Lippincott Williams \& Wilkins; 2007. p. 930-961.

[11] Ronco C, Bellomo R, Kellum JA. Acute kidney injury. Contrib Nephrol 2007;156:340-353.

[12] Keene WR, Jandl JH. The sites of hemoglobin catabolism. Blood 1965;26:705-719.

[13] Gorjipour F, Totonchi Z, Gholampour Dehaki M, et al. Serum levels of interleukin-6, interleukin-8, interleukin-10, and tumor necrosis factor- $\alpha$, renal function biochemical parameters and clinical outcomes in pediatric cardiopulmonary bypass surgery. Perfusion 2019;34:651-659.

[14] Blinder JJ, Goldstein SL, Lee VV, et al. Congenital heart surgery in infants: Effects of acute kidney injury on outcomes. J Thorac Cardiovasc Surg 2012;143:368-374.

[15] Sethi SK, Kumar M, Sharma R, Bazaz S, Kher V. Acute kidney injury in children after cardiopulmonary bypass: risk factors and outcome. Indian Pediatr 2015;52:223-226.

[16] Lee SH, Kim SJ, Kim HJ, Son JS, Lee R, Yoon TG. Acute kidney injury following cardiopulmonary bypass in childrenrisk factors and outcomes. Circ J 2017;81:1522-1527.

[17] Nadim MK, Forni LG, Bihorac A, et al. Cardiac and vascular surgery-associated acute kidney injury: the 20th International Consensus Conference of the ADQI (Acute Disease Quality Initiative) Group. J Am Heart Assoc 2018;7:e008834.

[18] Mao H, Katz N, Ariyanon W, et al. Cardiac surgery-associated acute kidney injury. Cardiorenal Med 2013;3:178-199.

[19] Kanji HD, Schulze CJ, Hervas-Malo M, et al. Difference between pre-operative and cardiopulmonary bypass mean arterial pressure is independently associated with early cardiac surgery-associated acute kidney injury. J Cardiothorac Surg 2010;5:71.

[20] Hirata Y. Cardiopulmonary bypass for pediatric cardiac surgery. Gen Thorac Cardiovasc Surg 2018;66:65-70.

[21] Alam S, Shalini A, Hegde R, Mazahir R. Acute kidney injury as a predictor of poor outcome post cardiopulmonary bypass in children. Int J Contemp Pediatr 2017;4:234-240.

[22] Massé L, Antonacci M. Low cardiac output syndrome: identification and management. Crit Care Nurs Clin North Am 2005;17:375-383.

[23] Hoffman TM, Wernovsky G, Atz AM, et al. Prophylactic intravenous use of milrinone after cardiac operation in pediatrics (PRIMACORP) study. Prophylactic Intravenous Use of Milrinone After Cardiac Operation in Pediatrics. Am Heart J 2002;143:15-21.

[24] Chandler HK, Kirsch R. Management of the low cardiac output syndrome following surgery for congenital heart disease. Curr Cardiol Rev 2016;12:107-111.

[25] Raina R, Sethi SK, Wadhwani N, Vemuganti M, Krishnappa V, Bansal SB. Fluid overload in critically Ill children. Front Pediatr 2018;6:306.

[26] Wang N, Jiang L, Zhu B, Wen Y, Xi XM. Fluid balance and mortality in critically ill patients with acute kidney injury: a multicenter prospective epidemiological study. Crit Care 2015;19:371.

[27] Hassinger AB, Wald EL, Goodman DM. Early postoperative fluid overload precedes acute kidney injury and is associated with higher morbidity in pediatric cardiac surgery patients. Pediatr Crit Care Med 2014;15:131-138.

[28] Delpachitra MR, Namachivayam SP, Millar J, Delzoppo C, Butt WW. A case-control analysis of postoperative fluid balance and mortality after pediatric cardiac surgery. Pediatr Crit Care Med 2017;18:614-622.

[29] Greenberg JH, Zappitelli M, Devarajan P, et al. Kidney outcomes 5 years after pediatric cardiac surgery: the TRIBEAKI study. JAMA Pediatr 2016;170:1071-1078.

[30] Cooper DS, Claes D, Goldstein SL, et al. Follow-up renal assessment of injury long-term after acute kidney injury (FRAIL-AKI). Clin J Am Soc Nephrol 2016;11:21-29.

[31] Slocum JL, Heung M, Pennathur S. Marking renal injury: can we move beyond serum creatinine? Transl Res 2012; 159:277-289.

[32] Villa P, Jiménez M, Soriano MC, Manzanares J, Casasnovas $P$. Serum cystatin $C$ concentration as a marker of acute renal dysfunction in critically ill patients. Crit Care 2005;9: R139-R143.

[33] Peco-Antić A, Ivanišević I, Vulićević I, et al. Biomarkers of acute kidney injury in pediatric cardiac surgery. Clin Biochem 2013;46:1244-1251.

[34] Yuan SM. Acute kidney injury after pediatric cardiac surgery. Pediatr Neonatol 2019;60:3-11.

[35] Basu RK, Wong HR, Krawczeski CD, et al. Combining functional and tubular damage biomarkers improves diagnostic precision for acute kidney injury after cardiac surgery. J Am Coll Cardiol 2014;64:2753-2762. Erratum in: J Am Coll Cardiol 2015;65:1158-1159.

[36] McCullough PA, Ostermann M, Forni LG, et al. Serial urinary tissue inhibitor of metalloproteinase-2 and insulin- 
like growth factor-binding protein 7 and the prognosis for acute kidney injury over the course of critical illness. Cardiorenal Med 2019;9:358-369.

[37] Sato E, Kamijo-Ikemori A, Oikawa T, et al. Urinary excretion of liver-type fatty acid-binding protein reflects the severity of sepsis. Ren Replace Ther 2017;3:26.

[38] Zappitelli M, Krawczeski CD, Devarajan P, et al. Early postoperative serum cystatin $\mathrm{C}$ predicts severe acute kidney injury following pediatric cardiac surgery. Kidney Int 2011;80: 655-662.

[39] Koyner JL, Garg AX, Shlipak MG, et al. Urinary cystatin C and acute kidney injury after cardiac surgery. Am J Kidney Dis 2013;61:730-738.

[40] Meersch M, Schmidt C, Hoffmeier A, et al. Prevention of cardiac surgery-associated AKI by implementing the KDIGO guidelines in high risk patients identified by biomarkers: the PrevAKI randomized controlled trial. Intensive Care Med 2017;43:1551-1561.

[41] Toda Y, Sugimoto K. AKI after pediatric cardiac surgery for congenital heart diseases-recent developments in diagnostic criteria and early diagnosis by biomarkers. $J$ Intensive Care 2017;5:49.

[42] Jetton JG, Rhone ET, Harer MW, et al. Diagnosis and treatment of acute kidney injury in pediatrics. Curr Treat Options Pediatr 2016;2:56-68.

[43] Ricci Z, Luciano R, Favia I, et al. High-dose fenoldopam reduces postoperative neutrophil gelatinase-associated lipocaline and cystatin C levels in pediatric cardiac surgery. Crit Care 2011;15:R160.

[44] Thomas MC, Harris DCH. Problems and advantages of continuous renal replacement therapy. Nephrology 2002;7: 110-114.

[45] Akoh JA. Peritoneal dialysis associated infections: an update on diagnosis and management. World J Nephrol 2012; 1:106-122.

[46] Gibney RT. Continous renal replacement therapy and intermittent hemodialysis in acute kidney injury: equivalent or complementary? J Thorac Dis 2016;8:2397-2399.

[47] Jander A, Tkaczyk M, Pagowska-Klimek I, et al. Continuous veno-venous hemodiafiltration in children after cardiac surgery. Eur J Cardiothorac Surg 2007;31:1022-1028.

[48] Namachivayam SP, Butt W, Millar J, Konstantinov IE, Nguyen C, d'Udekem Y. Early peritoneal dialysis and major adverse events after pediatric cardiac surgery: a propensity score analysis. Pediatr Crit Care Med 2019;20:158-116.

[49] Kwiatkowski DM, Menon S, Krawczeski CD, et al. Improved outcomes with peritoneal dialysis catheter placement after cardiopulmonary bypass in infants. $J$ Thorac Cardiovasc Surg 2015;149:230-236.

[50] Ryerson LM, Mackie AS, Atallah J, et al. Prophylactic peritoneal dialysis catheter does not decrease time to achieve a negative fluid balance after the Norwood procedure: a randomized controlled trial. J Thorac Cardiovasc Surg 2015; 149:222-228.

[51] Oh DJ. A long journey for acute kidney injury biomarkers. Ren Fail 2020;42:154-165.

[52] Fan PC, Chen CC, Chen YC, Chang YS, Chu PH. MicroRNAs in acute kidney injury. Hum Genomics 2016;10:29.

[53] Zhou D, Tan RJ, Fu H, Liu Y. Wnt/ $\beta$-catenin signaling in kidney injury and repair: a double-edged sword. Lab Invest 2016;96:156-167.

[54] Hattori Y, Kim H, Tsuboi N, et al. Therapeutic potential of stem cells from human exfoliated deciduous teeth in models of acute kidney injury. PLoS One 2015;10:e0140121. Erratum in: PLoS One 2015;10:e0143561.

[55] Herrera Sanchez MB, Bruno S, Grange C, et al. Human liver stem cells and derived extracellular vesicles improve recovery in a murine model of acute kidney injury. Stem Cell Res Ther 2014;5:124.

[56] Pickkers P, Heemskerk S, Schouten J, et al. Alkaline phosphatase for treatment of sepsis-induced acute kidney injury: a prospective randomized double-blind placebocontrolled trial. Crit Care 2012;16:R14. 